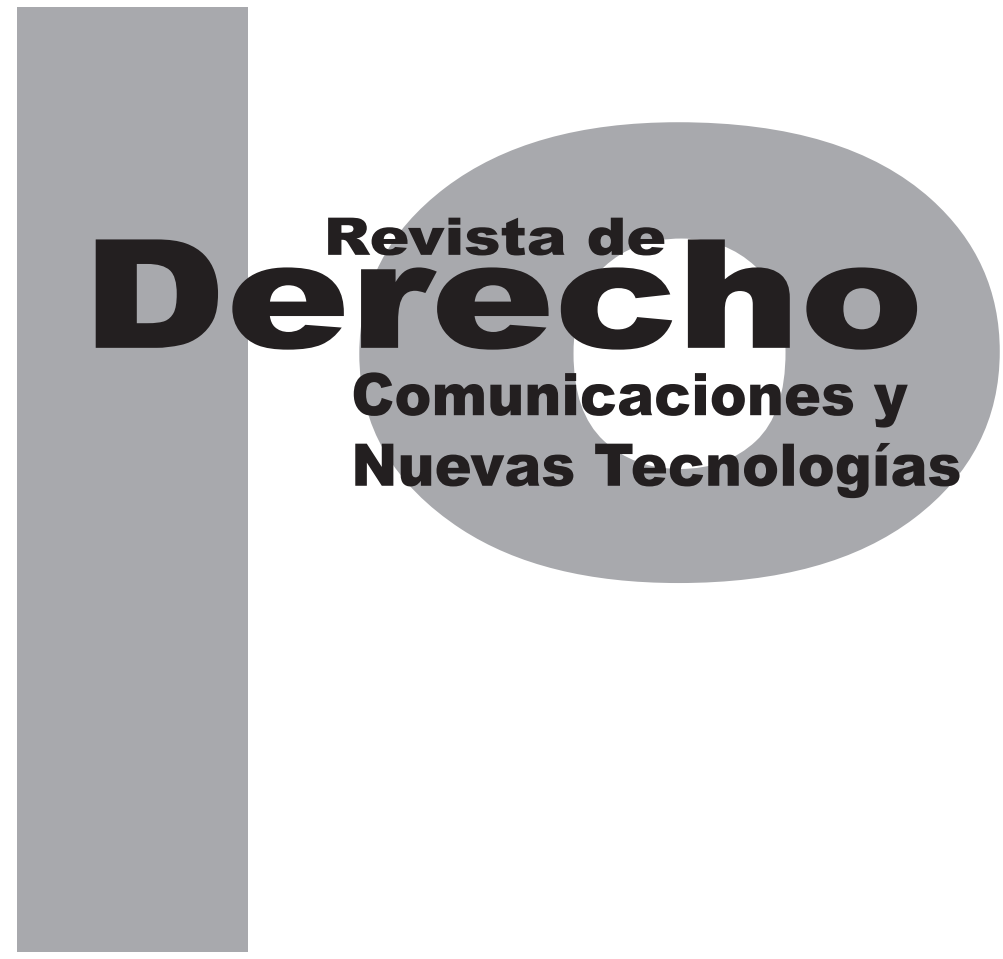

ACCESO A LA ÓRBITA DE LOS SATÉLITES geoestacionarios. Propuesta para un RÉgIMEN JURÍDICO ESPECIAL

\author{
Sylvana PeÑa SAFFon
}

Revisión de tema

Universidad de los Andes

Facultad de Derecho

Revista de Derecho, Comunicaciones y Nuevas Tecnologías

No.11, Enero - Junio de 2014. ISSN 1909-7786 


\section{Acceso a la órbita de los satélites geoestacionarios. Propuesta para un régimen jurídico especial}

Resumen

El texto tiene como objetivo estudiar las posibilidades de regulación jurídica de la órbita de los satélites geoestacionarios. El artículo estudia la problemática alrededor del acceso y uso de esta regulación con el fin de analizar los esfuerzos por reglamentar jurídicamente su uso. Expone, además, la necesidad de desarrollar puntualmente la propuesta del documento (A/AC.105/738, anexo III) aprobado como resolución por las Naciones Unidas, y vigente para el cumplimiento de los principios que rigen el derecho del espacio ultraterrestre, para concluir con una propuesta de reglamentación jurídica que busca poner fin a la problemática en torno al espacio.

Palabras clave: Órbita de los satélites geoestacionarios, recurso órbita-espectro, Unión Internacional de Telecomunicaciones, Régimen jurídico especial, Tratado de 1967.

\section{Geoestationary Earth orbit access. A proposal for a special legal regulation}

\section{Abstract}

The following text concerns the subject related to the geoestationary Earth orbit and its legal regulations. This particular orbit has been problematic in matters of access and usage, and the present text will study it, followed by the analysis of the existing efforts to regulate it. The necessity of developing a particular regulation from the current efforts will be shown, towards fulfilling the basic principles in Space Law. The text will conclude with a specific proposal to develop a special legal regulation that seeks to solve the problem concerning this particular spot in outer space.

Keywords: Geoestationary earth orbit, spectrum-orbit resource, International Telecommunication Union, Special legal regulation, 1967 Treaty.

\section{Acesso à órbita dos satélites geoestacionários. Proposta para um regime jurídico especial}

\section{Resumo}

O texto tem como objetivo estudar as possibilidades de regulação jurídica da órbita dos satélites geoestacionários. 0 artigo estuda a problemática ao redor do acesso e uso desta regulação com o fim de analisar os esforços por regulamentar juridicamente seu uso. Expõe, além disso, a necessidade de desenvolver pontualmente a proposta do documento ( $\mathrm{A}$ ) AC.105/738, anexo III) aprovado como resolução pelas Nações Unidas, e vigente para o cumprimento dos princípios que regem o direito do espaço ultra terrestre, para concluir com uma proposta de regulamentação jurídica que busca acabar com a problemática em torno do espaço.

Palavras-chave: Órbita dos satélites geoestacionários, recurso órbita/espectro, União Internacional de Telecomunicações, Regime jurídico especial, Tratado de 1967 


\section{SUMARIO}

Introducción - I. ASPECTOS TÉCNICOS Y CIENTÍFICOS - A. Satélites artificiales - B. Órbita de los satélites geoestacionarios - C. Espectro electromagnético y espectro radioeléctrico - D. Recurso órbita-espectro - II. RECUENTO HISTÓRICO - III. ASPECTOS JURÍDICOS - A. Principios generales: Resolución de 1963 y Tratado de 1967 - B. Declaración de Bogotá de 1976 - C. Consenso, confianza y cooperación: desarrollo progresivo del derecho internacional - 1. Artículo 2 del Tratado de 1967 y artículo 101 de la Constitución Política de Colombia - D. Unión Internacional de Telecomunicaciones - 1. Convenio de Montreux de 1965 - 2. Convenio de Málaga-Torremolinos de 1973 - 3. Convenio de Nairobi de 1982 - 4. Constitución de la UIT: Conferencia Plenipotenciaria de Minneapolis de 1998 - 5. Reglamento de Radiocomunicaciones - 5.1. Solicitud recurso órbita-espectro - 5.2. Procedimiento para la adjudicación de frecuencias - 5.3. Problemas del procedimiento - E. "Algunos aspectos relativos a la utilización de la órbita geoestacionaria", documento aprobado por la Subcomisión de Asuntos Jurídicos en su 39. ${ }^{\circ}$ período de sesiones (A/AC.105/738, anexo III) - IV. RÉGIMEN JURÍDICO ESPECIAL - A. Exposición - B. Desarrollo - C. Procedimiento - V. CONCLUSIONES - Referencias 


\title{
Acceso a la órbita de los satélites geoestacionarios. Propuesta para un régimen jurídico especial $^{1}$
}

\author{
Sylvana Peña Saffon ${ }^{2}$
}

Introducción

A través de la historia, la órbita de los satélites geoestacionarios ha sido objeto de grandes controversias. Desde que se descubrió su utilidad para desarrollar las telecomunicaciones globales, el acceso a posiciones orbitales a esta altura sobre la Tierra ha sido inequitativo. Los países desarrollados, con programas espaciales, han tenido mayores posibilidades de lanzar satélites a 35865 kilómetros sobre la línea ecuatorial de la Tierra. Para el resto de países el acceso es limitado. En 1945, Arthur C. Clarke, escritor británico, publicó su teoría sobre la órbita de los satélites geoestacionarios. En un documento explicaba cómo a una determinada altura sobre la línea ecuatorial de la Tierra podrían funcionar

Cómo citar este artículo: Peña Saffon, S. (Junio, 2014). Acceso a la órbita de los satélites geoestacionarios. Propuesta para un régimen jurídico especial. Revista de Derecho, Comunicaciones y Nuevas Tecnologías, 11.

2 Estudiante de décimo semestre de la Facultad de Derecho de la Universidad de los Andes. Monitora de la cátedra de la misma universidad, Derecho del Espacio Ultraterrestre, dictada por Alfredo Rey Córdoba, 2013-Il y 2014-I. satélites que formarían una red de comunicación global, obviando los problemas que para la época presentaban el telégrafo, el teléfono y la televisión. La importancia de este punto en el espacio ultraterrestre sería monumental.

Este artículo busca aproximarse, primero, a los aspectos técnicos alrededor de la problemática del acceso a la órbita de los satélites geoestacionarios. Será también necesario referirse a eventos históricos que cambiaron la concepción que se le dio, en un principio, a este punto en el espacio, para luego analizar los aspectos jurídicos relacionados. Por último, el texto presentará una propuesta que desarrolla lo enunciado en el documento "Algunos aspectos relativos a la utilización de la órbita geoestacionaria”, aprobado por la Subcomisión de Asuntos Jurídicos en su 39. ${ }^{\circ}$ período de sesiones (A/AC.105/738, Anexo III). El objetivo principal de este artículo es desarrollar una propuesta de un régimen jurídico especial con base en el documento A/ AC.105/738, resolución de las Naciones Unidas. El problema de investigación se centra, 
entonces, en formular esta propuesta, teniendo en cuenta la importancia del análisis sobre si el régimen especializado es necesario o no.

Para elaborar el texto se llevó a cabo una amplia investigación, y se analizaron cada uno de sus aspectos más importantes. Esto permitió formular una propuesta que busca poner en práctica los principios de las Naciones Unidas sobre el acceso a la llamada "órbita de los satélites geoestacionarios". La primera parte se referirá a los aspectos técnicos. La segunda, a los hechos históricos que tuvieron influencia en el uso de este recurso. En una tercera parte se expondrá la regulación tanto de las Naciones Unidas como de la Unión Internacional de Telecomunicaciones, para concluir con la propuesta de un régimen jurídico especial que desarrolla la resolución mencionada.

\section{ASPECTOS TÉCNICOS Y CIENTÍFICOS}

\section{A. Satélites artificiales}

Un satélite es un objeto que gira alrededor de otro cuerpo. El ejemplo más común es el de la Luna como satélite natural. La Luna es un cuerpo que gira alrededor de la Tierra. Los satélites artificiales, en cambio, son fabricados por el hombre y lanzados al espacio con el fin de que giren alrededor de la Tierra, o, en otros casos, alrededor de otros cuerpos celestes.

Los satélites tienen estructuras ligeras metálicas que les permiten girar alrededor de la Tierra. Tienen antenas que reciben y transmiten información desde y hacia la Tierra; paneles solares que los alimentan de energía; pequeñas antenas de telemetría y telecontrol que permiten controlar su movimiento en órbita desde la Tierra, y una caja central de carga útil que permite realizar la función específica para la que fue creado.

Cuando el satélite está en órbita apenas necesita elementos de sostén. Su estructura principal tiene dos misiones: al principio, resistir durante unos minutos la aceleración del cohete portador hasta el momento de la entrada en órbita; una vez en el espacio, mantener unidos los diferentes módulos del satélite y ofrecer una estructura rígida sobre la que puedan actuar esporádicamente los motorcitos de maniobra o de control de posición. (Satélites artificiales, 1973, p. 37)

Los satélites son enviados para cumplir diversas funciones: brindar telecomunicaciones alrededor de la Tierra, observar la Tierra en busca de desastres naturales, como huracanes, tsunamis, incendios. Si se identifican algunos de estos eventos, a través de los satélites se informa al país o países afectados. Cumplen la función, además, de pronosticar el clima, entre otros. En otras palabras, hay varios tipos de satélites: científicos, de comunicaciones, meteorológicos, de navegación, militares, etcétera.

\section{B. Órbita de los satélites geoestacionarios}

Si se tiene claro el concepto de satélite artificial, es más fácil acercarse al de órbita. La Real Academia Española define una órbita como la 
“trayectoria que, en el espacio, recorre un cuerpo sometido a la acción gravitatoria ejercida por Ios astros". Es decir, un cuerpo, ya sea natural o artificial, describe un camino alrededor de otro cuando este lo atrae con su fuerza gravitatoria. Si no hay un cuerpo que trace esta trayectoria, la órbita no existe.

Los satélites artificiales tienen la capacidad de orbitar la Tierra en la medida en que actúan sobre ellos las fuerzas centrifuga y la gravitacional de la Tierra. La Luna es el único satélite natural que orbita la Tierra. Sin embargo, los satélites artificiales abundan en el espacio, y describen diversos tipos de órbitas alrededor de la Tierra.

Las órbitas más comunes son las circulares, que describen un círculo alrededor de la Tierra, ya sea sobre el Ecuador o sobre cualquier otro punto del planeta. Las órbitas elípticas, en cambio, tienen un punto máximo de alejamiento de la Tierra, y un punto mínimo de acercamiento, conocidos como apogeo y perigeo. Si el satélite se encuentra más cerca de la Tierra, girará a gran velocidad. A medida que los satélites se alejan, la velocidad a la que orbitan disminuye.

Específicamente, la órbita de los satélites geoestacionarios es la que describe un objeto a 35865 kilómetros, aproximadamente, sobre la línea ecuatorial de la Tierra. A esta órbita se le llama también geosincrónica, pues el período nodal del satélite -es decir, el tiempo que tarda en recorrer una circunferencia- dura, exactamente, 23 horas, 56 minutos y 4 segundos, lo que significa que gira alrededor de la Tierra a su misma velocidad. Esto es importante, pues el satélite está siempre en el mismo punto con respecto a la Tierra.

Esta órbita ha sido fundamental para el desarrollo de las telecomunicaciones, pues el satélite siempre señala a un mismo punto, y su antena receptora en la Tierra mantiene comunicación permanente.

\section{B. Espectro electromagnético y espectro radioeléctrico}

El espectro electromagnético es el "conjunto de ondas electromagnéticas que existen en el universo, ordenadas en función de sus frecuencias o longitudes de onda, o de la energía que transportan" (Portal del Estado Colombiano). Dentro de estas ondas se encuentran las radioeléctricas, que pueden ser largas, medias, cortas, ultracortas o microondas, luz infrarroja, luz visible, luz ultravioleta, rayos $\mathrm{X}$, rayos gamma y rayos cósmicos.

El espectro radioeléctrico hace parte del electromagnético. Las ondas son perturbaciones que transportan energía, y se propagan a través del espacio. La frecuencia de las ondas radioeléctricas se ha fijado por debajo de los tres mil gigahercios, y se propagan sin una guía artificial. Es decir, la velocidad de repetición de la onda es de una vez por segundo. Estas son las ondas que se utilizan en las radiocomunicaciones.

Las radiocomunicaciones son telecomunicaciones que se transmiten a través de ondas radioeléctricas. Las telecomunicaciones son las transmisiones, emisiones o recepciones de 
cualquier signo, escrito, señal, imagen, sonido o información por medio de hilos, medios ópticos, radioelectricidad, entre otros.

\section{Recurso órbita-espectro}

Los satélites funcionan desde una posición orbital determinada en el espacio, y transmiten información a través de frecuencias. El recurso órbita-espectro (ROE) es "el recurso natural constituido por la órbita de los satélites geoestacionarios u otras órbitas de satélites, y el espectro de frecuencias radioeléctricas atribuido o adjudicado a los servicios de radiocomunicaciones por satélite por la Unión Internacional de Telecomunicaciones" (Organización de los Estados Americanos [OEA], Decisión 395). Es decir, un satélite funciona solo si usa el ROE.

\section{RECUENTO HISTÓRICO}

La carrera espacial comenzó en 1957 con el lanzamiento del primer satélite artificial por parte de la Unión Soviética: el Sputnik I. La posibilidad de lanzar satélites al espacio fue analizada durante varios años, y las conjeturas eran infinitas. Una de las teorías que probó ser definitiva en cuanto al lanzamiento de satélites y de su funcionamiento geoestacionario fue la de Arthur C. Clarke.

Clarke, autor británico, escribió en 1945 -doce años antes de la primera incursión en el espacio exterior- un artículo técnico, "Extra-terrestrial Relays", en el que señalaba la posibilidad de usar en el espacio una órbita de satélites geoestacionarios. Para ese entonces, tanto la comunicación telefónica y telegráfica, como las emisiones por televisión, eran complejas. Había muchos obstáculos, y las estaciones de televisión solo tenían la capacidad de llevar la imagen a pocos kilómetros de distancia. Fue entonces necesario un sistema de transmisión global que solucionase estas dificultades.

En ese momento, la ciencia incursionaba en la construcción de cohetes; esta podía ser la solución. Clarke sostenía que si un cohete alcanza cierta velocidad, puede abandonar la Tierra, sin posibilidad de volver a entrar. Giraría entonces alrededor de la Tierra; es decir, la orbitaría. Más adelante se construirían cohetes manejados por radio que tendrían la posibilidad de transmitir, desde más allá de la atmósfera, información científica a la Tierra. Por último, se construirían cohetes tripulados por humanos que contarían con la fuerza suficiente para romper su órbita y regresar a la Tierra.

Las posibilidades de orbitar alrededor de la Tierra podrían ser infinitas, desde circulares, a elípticas, a alturas variadas sobre la tierra. Mientras la órbita se encontrara más cerca de la Tierra la velocidad sería mayor. Mientras más se alejara, la velocidad disminuiría en la medida en que la gravedad también disminuye.

Clarke señaló que una órbita alrededor de la Tierra con un radio de 42000 kilómetros, vuelve a un mismo punto en un período de 24 horas. $\mathrm{Si}$ esta órbita se sitúa sobre la línea del ecuador, explicó Clarke, los objetos allí colocados giran al mismo tiempo que la Tierra, y, por lo tanto, 
permanecen en el mismo punto con relación a la Tierra, lo que permite la implementación de sistemas globales de comunicación que -resaltó Clarke-solucionarían todos los problemas de transmisión de información (1945).

\section{ASPECTOS JURÍDICOS}

\section{A. Principios generales: Resolución de 1963 y Tratado de 1967}

El primer documento aprobado por la Organización de las Naciones Unidas (ONU) sobre espacio ultraterrestre fue la "Declaración de los principios jurídicos que deben regir las actividades de los Estados en la exploración y utilización del espacio ultraterrestre", aprobada por la Asamblea General de la ONU en Resolución 1962 (XVIII) del 13 de diciembre de 1963. Este documento fue el marco fundamental para el esbozo y aprobación del "Tratado sobre los principios que deben regir las actividades de los Estados en la exploración y utilización del espacio ultraterrestre, incluso la Luna y otros cuerpos celestes", aprobado por la Asamblea General en Resolución 2222 (XXI) del 19 de diciembre de 1966.

La Resolución de 1963 enunció los principios que serían parte fundamental de los tratados, y demás documentos, aprobados por las Naciones Unidas para la regulación del espacio ultraterrestre, tales como la exploración en provecho de la humanidad, la igualdad de acceso, la no apropiación, el uso pacífico, la responsabilidad por daños, la asistencia mutua, el registro de objetos espaciales, entre otros.
Para efectos del tema bajo estudio es menester mencionar que el principio número 3 de la Resolución de 1963 señala que "el espacio ultraterrestre y los cuerpos celestes no podrán ser objeto de apropiación nacional mediante reivindicación de soberanía, mediante el uso y la ocupación, ni de ninguna otra manera".

Más adelante, el Tratado de 1967 establece como principios generales del espacio ultraterrestre la cooperación, la no apropiación y el uso pacífico del espacio. En el artículo I se señala que

la exploración y utilización del espacio ultraterrestre, incluso la Luna y otros cuerpos celestes, deberán hacerse en provecho y en interés de todos los países, sea cual fuere su grado de desarrollo económico y científico, e incumben a toda la humanidad. El espacio ultraterrestre, incluso la Luna y otros cuerpos celestes, estará abierto para su exploración y utilización a todos los Estados sin discriminación alguna en condiciones de igualdad y de conformidad con el derecho internacional, y habrá libertad de acceso a todas las regiones de los cuerpos celestes. El espacio ultraterrestre, incluso la Luna y otros cuerpos celestes, estarán abiertos a la investigación científica, y los Estados facilitarán y fomentarán la cooperación internacional en dichas investigaciones.

El artículo II, por su parte, aclara que "el espacio ultraterrestre, incluso la Luna y otros cuerpos celestes, no podrá ser objeto de apropiación nacional por reivindicación de soberanía, uso u ocupación, ni de ninguna otra manera". Es decir, el principio de no apropiación supone que en el espacio ultraterrestre no hay soberanía de ningún 
Estado. Ulrike Bohlmann (2005) subraya: "The freedom to use outer space is only conceivable if territorial sovereignty is excluded. Accordingly, the principle of non-appropriation has to be understood as a necessary corollary to the general freedom to use outer space" (p. 221).

Para que la intención de libertad general en el espacio se realice, no es posible inclinarse por derechos exclusivos de unos pocos. Este principio reafirma el derecho igual para todos los países sobre el espacio y lo que en él se encuentre. Es, además, importante estudiar los conceptos de "apropiación nacional" y "apropiación por uso u otros medios".

El primer concepto no solo cobija la apropiación en los términos de la soberanía nacional, sino en los de apropiación por entidades privadas. Frente a esto, Ulrike se remite a los documentos preparatorios del "Tratado sobre el espacio", y aclara lo siguiente: "[...] This interpretation is confirmed by recourse of the preparatory work of the Outer Space Treaty as supplementary means of interpretation. In fact, the term "national' was not used in the sense as opposed to private but as opposed to international" ( $p$. 223). El segundo concepto no se ha discutido ampliamente, pero se cree que el significado de "apropiación por cualquier otro medio" busca cubrir cualquier espectro de actuación que pueda constituir un acto de apropiación.

El artículo III, por último, establece que

Ios Estados parte del Tratado deberán realizar sus actividades de exploración y utilización del espacio ultraterrestre, incluso la Luna y otros cuerpos celestes, de conformidad con el derecho internacional, incluida la Carta de las Naciones Unidas, en interés del mantenimiento de la paz y la seguridad internacionales, y del fomento de la cooperación y la comprensión internacionales.

Estos principios generales regulan todas las actividades relacionadas con el espacio ultraterrestre, y todos los cuerpos y objetos que en él se encuentran. Estos principios aplican, por ende, también al régimen que debe regular las actividades realizadas en la órbita de los satélites geoestacionarios y el acceso a la misma.

\section{B. Declaración de Bogotá de 1976}

Casi diez años después de la expedición del Tratado marco sobre el derecho del espacio, los países ecuatoriales, es decir, los países cuyo territorio es atravesado por la línea del ecuador -Ecuador, Brasil, Indonesia, Gabón, República del Congo, República Democrática del Congo, Zaire (posteriormente República Democrática del Congo), Uganda, Kenia, Somalia y Colombia-, se reunieron en Bogotá para estudiar las implicaciones de la órbita geoestacionaria.

En esta reunión se señaló que, en un punto sobre la Tierra, a 35865 kilómetros, se produce un fenómeno gravitacional en el que un objeto allí puesto se comporta de cierta forma. Por esto, este punto en el espacio no hacía parte del espacio exterior. Debido a esto, la órbita geoestacionaria debía ser considerada como parte de los territorios ecuatoriales y se debía ejercer allí su soberanía. Así, teniendo en cuenta que la órbita es un recurso natural, los países ecuato- 
riales reivindicaron soberanía sobre el mismo. Además, se argumentaba que en la medida en que no existía una delimitación sobre el espacio, no se podía establecer que la órbita hiciera parte de este y, por ende, el Tratado de 1967 no tenía que ser aplicado.

Excepto Brasil, que firmó como observador, se suscribió la Declaración de Bogotá, para luego ser expuesta en la Asamblea General de las Naciones Unidas.

\section{Consenso, confianza y cooperación: desarrollo progresivo del derecho internacional}

Una vez estudiados los principios generales, y el documento de la Declaración de Bogotá, es necesario analizar el proceso legislativo que origina las normas internacionales sobre derecho del espacio, y la obligatoriedad de estas.

Los asuntos relacionados con el espacio ultraterrestre son reglamentados por la Organización de las Naciones Unidas, específicamente, por la Comisión sobre la Utilización del Espacio Ultraterrestre con Fines Pacíficos (Copuos). El derecho internacional, en general, tiene aplicabilidad sobre las actividades que se lleven a cabo en el espacio ultraterrestre. La Resolución de 1963 así lo expresa:

Las actividades de los Estados en materia de exploración y utilización del espacio ultraterrestre deberán realizarse de conformidad con el derecho internacional, incluida la Carta de las Naciones Unidas, en interés del mantenimiento de la paz y la seguridad internacionales y del fomento de la cooperación y la comprensión internacionales. (Núm. 4)

A saber, los propósitos de las Naciones Unidas expresados en su artículo 1 de la Carta se refieren a mantener la paz y la seguridad nacionales, fomentar las relaciones de amistad entre las naciones, resolver problemas mediante la cooperación internacional y fungir como centro donde estos propósitos se puedan llevar a cabo.

Asimismo, el Tratado de 1967 remite a ello, al señalar que "un Tratado sobre los principios que deben regir las actividades de los Estados en la exploración y utilización del espacio ultraterrestre, incluso la Luna y otros cuerpos celestes, promoverá los propósitos y principios de la Carta de las Naciones Unidas".

Así, se entiende que el derecho del espacio ultraterrestre surge como parte del derecho internacional en el seno de las Naciones Unidas, y se crea como un derecho que busca preservar la paz y seguridad internacional así como el fomento de la cooperación. Este derecho encuentra sus orígenes en la Resolución de 1963, que sentó los fundamentos para la aprobación del Tratado de 1967.

Las decisiones que toma la Subcomisión de Asuntos Jurídicos del Copuos con el fin de aprobar resoluciones se diferencia del resto de decisiones de las Naciones Unidas en un aspecto fundamental: se toman por consenso, no por votación. ${ }^{3}$ Por consenso se entiende un acuerdo

3 En la Asamblea de las Naciones Unidas, las resoluciones pueden ser aprobadas por mayoría, aun cuando se busca el consenso, también para su aprobación. 
general sobre un tema en particular. Esto tendrá una repercusión importante en la obligatoriedad, tanto de las resoluciones, como de los tratados.

A propósito del consenso, Aldo Armando Cocca (1991) aclaró lo siguiente:

En el consenso se agrega el convencimiento de que es una actitud necesaria. Si se trata de una solución legal a un problema dado, el consenso es, por lo tanto, la convicción sobre la conveniencia o acierto de una proposición dada. Por ello, el consenso va más allá del voto por mayoría o por unanimidad. Ante el voto puede darse una elección por alternativa o por exclusión [...] En el consenso, ese común sentir supone la aceptación de la opción en todos sus alcances y además un sentimiento, a su respecto, que es común por parte de quienes la eligen. (p. 48)

El consenso, por tanto, no denota un acuerdo total. Implica, más bien, el no ejercicio de la oposición, pues es clara, como bien lo dice Cocca, "la aceptación de la opción en todos sus alcances".

Todas las resoluciones que aprueban la Subcomisión Jurídica y el Copuos surgen del consenso; luego son aprobadas como resoluciones o tratados en la Asamblea General. Por lo tanto, la obligatoriedad coercitiva de las normas es relativa. En el derecho internacional, las únicas normas de derecho positivo son los tratados. Las resoluciones se consideran soft law, "derecho blando". Sin embargo, se ha propuesto que las normas que se expiden por consenso sean consideradas, también, derecho positivo.
Cuando una decisión se toma por consenso, se genera, como consecuencia, una creciente confianza. Si todos aprobaron la decisión -así algunos no hubieran estado de acuerdo-, se confía en que todos cumplirán con sus preceptos. Esto llevará a dar cumplimiento al principio de cooperación estipulado en la Carta de las Naciones Unidas y en el Tratado de 1967, en la medida en que la exploración y utilización del espacio se llevará a cabo en provecho e interés de todos, razón por la cual se dará cumplimiento al principio de cooperación estipulado en la Carta de las Naciones Unidas y en el Tratado de 1967. Es decir, los Estados cumplirán con lo que se apruebe por consenso, no porque sea obligatorio, sino porque todos lo aprobaron. Por lo tanto, el cumplimiento tanto de tratados como de resoluciones debería darse independientemente de la firma o ratificación de los mismos. En el caso de que un Estado apruebe un asunto por consenso, pero ejerza oposición continua frente a uno de los puntos, el valor obligatorio de la costumbre no se materializa.

Colombia firmó el Tratado de 1967. Se opuso constantemente a la interpretación que se le dio al artículo II en cuanto a la órbita de los satélites geoestacionarios respecto de ejercer soberanía. Sin embargo, no ejerció oposición constante contra el artículo en sí. Por esto, sin importar la no ratificación del tratado, Colombia está obligada a cumplir con los preceptos de la norma. 


\section{Artículo II del Tratado de 1967 y artículo 101 de la Constitución Política de Colombia}

Ya hemos visto cómo la declaración de Bogotá desafió el artículo 2 del Tratado de 1967, que consagra la no apropiación del espacio ultraterrestre. La reivindicación de soberanía que proclaman los países ecuatoriales - a excepción de Brasil- viola la estipulación de no apropiación, pues los países consideran como propio el segmento de la órbita de los satélites geoestacionarios que suprayace cada uno de sus territorios. En el caso de Colombia, ese segmento se encuentra entre los grados 75 34' 57' W - 70 02' 37'.3W oeste (Instituto Agustín Codazzi, 1981).

De la reivindicación de la soberanía surgen los siguientes problemas: en primer lugar, la órbita de los satélites geoestacionarios hace parte del espacio ultraterrestre. ${ }^{4}$ Como tal, le aplican todos los principios y tratados que sobre él se refieran. Como se vio, el artículo II del Tratado de 1967 prohíbe expresamente la apropiación por reivindicación de soberanía o cualquier otro medio. Así las cosas, la reivindicación viola un tratado internacional, y, por lo tanto, va en contra de la Convención de Viena y el principio pacta sunt servanda, "lo pactado obliga".

En segundo lugar, la terminología usada por los países que reivindican la soberanía es errónea, pues se refiere a algo que no existe. La declara-

4 A pesar de que no hay consenso al respecto, en la práctica los Estados aceptan que cien kilómetros, perigeo mínimo de un satélite, es el límite entre el espacio aéreo en donde se ejerce soberanía, y el espacio ultraterrestre en el cual no se ejerce. ción de Bogotá se refiere a la "órbita geoestacionaria". Como se vio, las órbitas solo existen si un objeto las describe. Sin un objeto, la órbita deja de existir. Este punto en el espacio, por tanto, no puede ser físicamente apropiable. Es erróneo entonces referirse a la "órbita geoestacionaria"; por esto, en este artículo nos hemos referido a la "órbita de los satélites geoestacionarios". A pesar de que los segmentos de la circunferencia que describen los satélites a 35 865 kilómetros de altura sobre la Tierra no varían respecto de un punto del planeta, sí varían respecto de su posición en el espacio, pues giran alrededor de la Tierra y no están nunca en un mismo punto.

Ahora bien, en la declaración se resalta que la órbita no está en el espacio ultraterrestre. Puesto que el fenómeno que se produce en el punto señalado sobre los objetos es resultado, en parte, de la fuerza que ejerce la Tierra, los firmantes señalaron que este punto no debe considerarse parte del espacio ultraterrestre. Sin embargo, no es lógico estipular que, debido a la utilidad que este punto ofrece, deba considerarse ajeno al espacio ultraterrestre. Cada objeto que describe alguna de las órbitas señaladas se comporta de manera diferente, teniendo en cuenta la fuerza que ejerce la Tierra sobre ellos. El hecho de que sea un punto de especial utilidad para las telecomunicaciones no lleva a que se puedan violar los preceptos internacionales pactados.

Es cierto, no se ha establecido, puntualmente, un límite superior al espacio ultraterrestre. Sin embargo, se ha podido deducir que este es infinito y, por lo tanto, todo lo que se encuentre 
más allá del espacio aéreo de la Tierra es parte del espacio.

De acuerdo con la declaración de Bogotá, la Constitución Política de Colombia, en su artículo 101, señala que

también son parte de Colombia, el subsuelo, el mar territorial, la zona contigua, la plataforma continental, la zona económica exclusiva, el espacio aéreo, el segmento de la órbita geoestacionaria, el espectro electromagnético y el espacio donde actúa, de conformidad con el Derecho Internacional o con las leyes colombianas a falta de normas internacionales.

Así como en la declaración, en la Constitución se evidencia una imprecisión técnica importante. Para empezar, el artículo aclara que estos lugares son parte del territorio colombiano "de conformidad con el Derecho Internacional”. El Tratado de 1967 niega la posibilidad de la apropiación del espacio ultraterrestre. La norma de derecho internacional regula el espacio ultraterrestre, espacio donde se encuentra la órbita de los satélites geoestacionarios; por esto, no es procedente la norma nacional que proclama soberanía sobre un punto que se entiende propiedad común de la humanidad.

\section{Unión Internacional de Telecomunicaciones}

Se ha establecido el equívoco de la proclamación hecha por los países ecuatoriales y, más adelante, por Colombia en su Constitución. Ahora se analizará la reglamentación del uso y acceso a la órbita de los satélites geoestacio- narios de la Unión Internacional de Telecomunicación (UIT), teniendo en cuenta el concepto de recurso órbita-espectro (ROE).

La UIT es un organismo especializado de las Naciones Unidas que regula todo lo relacionado con tecnologías de información y comunicación. Es el organismo encargado de asignar frecuencias del espectro radioeléctrico, y posiciones orbitales alrededor de la Tierra. Es, por extensión, el encargado de asignar las posiciones orbitales y las frecuencias que se habilitan en la órbita de los satélites geoestacionarios. Tres convenios importantes ayudarán a entender cuál ha sido la evolución de la asignación de frecuencias y posiciones orbitales.

\section{Convenio de Montreux de 1965}

Para 1965, año de expedición del Convenio de Montreux, la UIT ya reglamentaba un uso racional del espectro de frecuencias radioeléctricas para la asignación de frecuencias a quienes lo solicitaran, siempre bajo el entendido de que son limitadas. Sin embargo, en el convenio no se mencionaba la órbita de los satélites geoestacionarios:

Los Miembros y Miembros asociados reconocen la conveniencia de limitar el número de las frecuencias y el espacio del espectro utilizados al mínimo indispensable para asegurar de manera satisfactoria el funcionamiento de los servicios necesarios. A tales fines, será conveniente que se apliquen, a la mayor brevedad, los adelantos técnicos más recientes. (Art. 46) 


\section{Convenio de Málaga-Torremolinos} de 1973

Casi diez años después de suscrito el Convenio de Montreux, con el Convenio de Málaga-Torremolinos se hace evidente la importancia de la órbita de los satélites geoestacionarios. Ahora no solo se menciona, sino que se le atribuyen características especiales, que marcan una gran diferencia en el uso que se le da con respecto de todos los otros puntos accesibles en el espacio:

1. Los Miembros procurarán limitar el número de frecuencias y el espectro utilizado al mínimo indispensable para asegurar el funcionamiento satisfactorio de los servicios necesarios. A tales fines, se esforzarán por aplicar, a la mayor brevedad, los adelantos técnicos más recientes.

2. En la utilización de bandas de frecuencias para las radiocomunicaciones espaciales, los Miembros tendrán en cuenta que las frecuencias y la órbita de los satélites geoestacionarios son recursos naturales limitados que deben utilizarse en forma eficaz y económica para permitir el acceso equitativo a esta órbita y a esas frecuencias a los diferentes países o grupos de países, según sus necesidades y los medios técnicos de que dispongan, de conformidad con lo establecido en el Reglamento de Radiocomunicaciones. (Art. 33, negrilla fuera del texto)

Es claro, entonces, que el recurso órbita-espectro que conforman las frecuencias radioeléctricas y la órbita de los satélites geoestacionarios es, ante todo, un recurso natural limitado. Más si se tiene en cuenta que las condiciones físicas que allí se dan son únicas en el universo, y se generan por fenómenos físicos naturales con frecuencias y espacios limitados para ubicar satélites.

Se atribuye además un uso eficaz y económico a este recurso natural limitado, que pone de presente la necesidad de que su uso se optimice para aprovecharlo al máximo, tal como lo establece el primer numeral del artículo 33 del convenio. El número de frecuencias y espectro utilizado debe ser el menor posible, con el fin de que los servicios puedan funcionar de la mejor manera posible, y de forma económica.

El uso de este recurso debe hacerse de forma eficaz y económica para que haya un acceso equitativo a él, según se presenten necesidades y se cuenten con los medios para llevarlo a cabo. Es importante resaltar el uso de la palabra "equitativo". Se refiere a un elemento encargado de igualar las desigualdades, diferente del significado de "igualitario", que equivale al tratamiento igual de todas las partes.

\section{Convenio de Nairobi de 1982}

En 1982, luego de la Declaración de Bogotá, se expidió una nueva convención que buscaba mediar entre las proclamaciones de los países ecuatoriales y la oposición del resto del mundo:

1. Los Miembros procurarán limitar el número de frecuencias y el espectro utilizado al mínimo indispensable para asegurar el funcionamiento satisfactorio de los servicios necesa- 
rios. A tales fines, se esforzarán por aplicar, a la mayor brevedad, los adelantos técnicos más recientes.

2. En la utilización de bandas de frecuencias para las radiocomunicaciones espaciales, los Miembros tendrán en cuenta que las frecuencias y la órbita de los satélites geoestacionarios son recursos naturales limitados que deben utilizarse en forma eficaz y económica, de conformidad con lo establecido en el Reglamento de Radiocomunicaciones, para permitir el acceso equitativo a esta órbita y a esas frecuencias a los diferentes países o grupos de países, teniendo en cuenta las necesidades especiales de los países en desarrollo y la situación geográfica de determinados países. (Art. 33, negrilla fuera del texto)

Como se ve, la mayor parte del artículo no se modifica. Sin embargo, se agrega que, para que haya acceso equitativo, deben tenerse en cuenta las "necesidades especiales de los países en desarrollo y la situación geográfica de determinados países". Para los países ecuatoriales era claro que la última frase adicionada se refería a ellos. El resto de países no aceptó esta postura.

\section{Constitución de la UIT: Conferencia Plenipotenciaria de Minneapolis de 1998}

En 1998, en la Conferencia Plenipotenciaria de Minneapolis, se modificó el párrafo 196.2 del artículo 44 de la Constitución de la UIT. Allí, la órbita de los satélites geoestacionarios es entendida de la siguiente manera:

En la utilización de bandas de frecuencias para las radiocomunicaciones, los Estados miem- bros tendrán en cuenta que las frecuencias y la órbita de los satélites geoestacionarios son recursos naturales limitados que deben utilizarse en forma racional, eficaz y económica, de conformidad con lo establecido en el Reglamento de Radiocomunicaciones, para permitir el acceso equitativo a esta órbita y a esas frecuencias a los distintos países o grupos de países, teniendo en cuenta las necesidades especiales de los países en desarrollo y la situación geográfica de determinados países. (Negrilla fuera del texto)

Lo estipulado en el Convenio de Nairobi, de 1982, pasa ahora a estar incluido en la Constitución de la UIT, en relación con las características propias de la órbita de los satélites geoestacionarios. Sin embargo, sobresale un nuevo requisito: el uso racional. Es decir, además de ser un recurso natural limitado, que debe usarse de forma eficaz y económica, para permitir el acceso equitativo se debe adjudicar de acuerdo con la razón, con el fin de que su uso sea equitativo.

\section{Reglamento de Radiocomunicaciones}

El Reglamento de Radiocomunicaciones de la UIT regula el uso y asignación de frecuencias radioeléctricas, así como el proceso de coordinación que se tiene que llevar a cabo para acceder a una determinada posición orbital. Es importante analizar el procedimiento que se lleva a cabo para la efectiva asignación de frecuencias y posiciones orbitales en lo que respecta a la órbita de los satélites geoestacionarios y, por ende, a los sistemas de telecomunicaciones. 
Antes de esto, cabe aclarar que todos los países del mundo son miembros de la UIT, y que todos ratificaron el tratado internacional que contiene el Reglamento de Radiocomunicaciones, incluido Colombia. Así, toda la regulación que determina el acceso a la órbita de los satélites geoestacionarios aplica para nuestro país.

\subsection{Solicitud de recurso órbita-espectro}

Cuando un país busca establecer un sistema satelital de telecomunicaciones, que implica el uso del ROE, debe notificar a la Junta del Reglamento de Radiocomunicaciones -antes, Junta Internacional de Registro de Frecuencias- de la UIT, por lo menos cinco años antes de que el sistema comience a funcionar, toda la información del sistema y de los satélites que lo conformarán. Esta notificación se entiende como una solicitud formal ante la UIT, con el fin de que se inscriba, para un país solicitante, la adjudicación de una posición orbital y la frecuencia radioeléctrica, elementos indispensables para que opere un sistema de telecomunicaciones. Es decir, se asigna el derecho de uso de una porción del ROE. ${ }^{5}$ Esta solicitud se debe notificar a todos los miembros de la UIT; luego se inscribe en el Registro Internacional de Frecuencias.

5 Se recurre a la palabra "uso", tanto de las posiciones orbitales, como de las frecuencias radioeléctricas, pues no hay soberanía en el espacio ultraterrestre, no hay derechos de dominio, y solo se autoriza su uso por un tiempo determinado.

\subsection{Procedimiento para la adjudicación de frecuencias}

Para que una frecuencia se adjudique a una administración, debe presentar para estudio toda la información relevante a la Oficina de Radiocomunicaciones de la UIT. Una vez presentada la información se llevará a cabo un proceso consistente en los pasos de publicación, coordinación, notificación e inscripción. Desde el momento en que la información es presentada para publicación, las administraciones tienen siete años para el inicio de la operación del sistema, pues, de lo contrario, la solicitud de publicación caduca, y se pierde la prioridad en el proceso.

Publicación anticipada: La sección I del artículo 9 del Reglamento señala que la administración debe dar a la Oficina de Radiocomunicaciones una descripción general del sistema, para que esta se publique en la Circular Internacional de Información sobre Frecuencias. En la información se debe dar la posición orbital de la que se piensa hacer uso, la frecuencia electromagnética, las características del o los satélites -como el nombre, las características físicas, el tiempo aproximado de desintegración o reingreso, los parámetros orbitales básicos, etc-, la naturaleza del servicio que prestará, etcétera.

Coordinación: A pesar de que el procedimiento de coordinación no siempre es necesario, lo es cuando se solicita el ROE para la órbita de los satélites geoestacionarios. La sección II del mencionado artículo establece que la coordinación se lleva a cabo para encontrar una solución a las interferencias que se puedan presentar en 
las transmisiones de la información, entre un satélite en órbita y el satélite que se quiere colocar, o entre dos sistemas o satélites que estén iniciando el proceso de solicitud de asignación del recuso órbita-espectro. Es menester mencionar que las interferencias se pueden presentar por la utilización de la misma frecuencia, o porque las antenas están direccionadas a la misma posición.

A través de la publicación anticipada, las administraciones pueden determinar si el nuevo uso de una posición orbital y de frecuencia radioeléctrica generará interferencias en sus satélites operantes, o en los que pretenden llevar a órbita. Las administraciones podrán presentar una solicitud de coordinación con las administraciones necesarias, administraciones con las que es necesario cooperar, pues se pueden llegar a producir interferencias con los satélites que se quieren poner en órbita, anexando la información, de acuerdo con los apéndices 4 y 5 del Reglamento.

Una vez se recibe la solicitud, cada administración deberá examinar sus interferencias, y comunicar, tanto a las administraciones dentro del proceso, como a la Oficina de Radiocomunicaciones - dentro de un plazo de cuatro meses-, un acuerdo o sugerencia de solución. Para resolver esto, se pueden celebrar reuniones, y recurrir a cualquier mecanismo, como la correspondencia, entre otros. Los resultados y las modificaciones se deben comunicar a la Oficina. Cabe resaltar que este proceso es largo y puede tomar años.
Si no se llega a un acuerdo, la administración interesada debe aplazar su notificación de asignación. Transcurridos seis meses, la administración puede presentar la notificación ante la Oficina, la cual evaluará las posibles interferencias. Si la Oficina considera que las interferencias no son graves, se llevará a cabo la notificación; de lo contrario, la solicitud de notificación será devuelta.

Notificación: Este procedimiento tiene como fin informar la frecuencia que se utilizará en el sistema de telecomunicaciones. Hasta que el sistema no opere, la asignación de frecuencias notificada se inscribirá provisionalmente.

Registro: En este último paso, la Oficina de Radiocomunicaciones evaluará las notificaciones; se considerarán en el orden de presentación. El contenido de la notificación será publicado por la Oficina, siempre que la información esté completa. Si se llega a una conclusión favorable sobre la notificación, la asignación de frecuencias se inscribirá en el Registro Internacional de Frecuencias. Si es desfavorable -pues persisten las interferencias-, se inscribirá, de manera informativa, hasta que sean eliminadas. Cumplidos estos pasos, se habrá concedido el derecho de uso de una posición orbital determinada, y de una frecuencia específica.

\subsection{Problemas del procedimiento}

En la práctica, el procedimiento de asignación del ROE ha sido eficaz. Sin embargo, en relación con el uso de la órbita de los satélites geoestacionarios, este procedimiento no siempre es 
el más apropiado para cumplir con los principios de equidad y racionalidad de los convenios mencionados.

El procedimiento refleja el principio de first come, first served, en otras palabras, "primero en tiempo, primero en derecho" o "primer llegado, primer servido". El país que primero registre ante la UIT una posición orbital y una frecuencia radioeléctrica, tendrá derecho de uso. Además, el procedimiento es largo, complejo y dispendioso. Para aquellos países con gran capacidad económica, y pioneros en materia espacial, no representa mayor problema. No pasa lo mismo con los países en vía de desarrollo, o con países sin programas espaciales fuertes. De cara al procedimiento de coordinación, es evidente la desventaja para este tipo de países.

El documento que se expone a continuación buscó resolver la problemática presentada por los países ecuatoriales, teniendo en cuenta, no solo los principios generales expuestos en el Tratado de 1967, sino los señalados por la UIT respecto del uso de la órbita de los satélites geoestacionarios.

\section{E. "Algunos aspectos relativos a la utilización de la órbita geoestacionaria", documento aprobado por la Subcomisión de Asuntos Jurídicos en su $39 .^{\circ}$ período de sesiones (A/AC.105/738, anexo III)}

Desde 1976, tras el largo debate de los países ecuatoriales, fue necesario llegar a un punto medio, en el que tuvieran cabida los principios rectores del derecho del espacio y se contara con el respaldo de todos los países, con el fin de evitar dificultades sobre los parámetros de la UIT en lo relativo a la asignación de frecuencias y posiciones orbitales.

En 2000, se aprobó una resolución titulada "Algunos aspectos relativos a la utilización de la órbita geoestacionaria", con una nueva tesis presentada por Alfredo Rey Córdoba, quien introdujo una nueva concepción de la órbita de los satélites geoestacionarios. La tesis de Rey Córdoba fue acogida por el Gobierno colombiano, y autorizada para ser presentada en el seno de la Subcomisión Jurídica.

Los países ecuatoriales proclamaban soberanía sobre los segmentos de órbita geoestacionaria que los suprayacían, y, por tanto, se consideraban los únicos autorizados para ubicar satélites allí, o dar permisos para que otros países lo hicieran. El resto de países se ciñó al Reglamento de Radiocomunicaciones, pues el acceso a las posiciones orbitales se daba de acuerdo con el principio de "quien llega primero, tiene prioridad".

Rey Córdoba partió de dos supuestos: primero, el artículo II del Tratado del 67 es de obligatorio cumplimiento, por lo tanto, no debe aceptarse la tesis de la soberanía en el espacio exterior. Segundo,

[...] no se puede negar que en el espacio ultraterrestre existe un lugar a los 35865 kilómetros sobre la línea ecuatorial de la Tierra, en el cual un objeto allí colocado se comporta con respecto a la Tierra de una manera diferente a como se comportaría en cualquier otro lugar de 
ese mismo espacio ultraterrestre. (Comunicación personal, 28 de enero de 2014)

Es decir, en palabras de Rey Córdoba, se concluye que este punto en el espacio debe tener una regulación jurídica diferente.

[...] Ese lugar debe tener un régimen jurídico distinto o especial con respecto al resto del espacio. Régimen jurídico que de acuerdo a los principios de equidad, que son normas de derecho positivo obligatorio, debería reflejarse en una modificación a los procesos de coordinación que se establecen en el Reglamento de Radiocomunicaciones cuando se dan unas circunstancias específicas, que se derivan del numeral 8 , literal a, del documento A/AC.105/738, anexo III, que finalmente en el literal d de ese mismo numeral remite a la UIT para que realicen esa reglamentación.

Este documento propone la constitución de un Régimen Jurídico Especial para regular el acceso a la órbita de los satélites geoestacionarios. El documento señala lo siguiente:

El acceso a las bandas de frecuencias distintas a las ya planificadas se rige actualmente por el principio de que "quien llega primero, tiene prioridad" ("first come, first served"). Este enfoque, si bien adecuado para los países desarrollados, puede poner en desventaja a los países en desarrollo, especialmente a los que aún no tienen acceso a dicha órbita. Los procedimientos de coordinación existentes que se aplican a las bandas no planificadas se han concebido para superar esa dificultad, pero no son necesariamente del todo satisfactorios. Es, pues, necesario facilitar el acceso al recurso órbitaespectro por parte de los países en desarrollo o de los países que aún están por acceder a este recurso, con respecto a los que ya lo utilizan. Es decir, asegurar un acceso equitativo entre los países que ya tienen acceso al recurso órbita-espectro y los que tratan de alcanzarlo. (Documento A/AC.105/738, anexo III, núm. 6, 2000)

El documento recuerda, de igual forma, que "las órbitas de los satélites y el espectro de frecuencias son recursos naturales limitados que deben utilizarse de forma racional, eficaz, económica y equitativa" (Núm. 7, literal a). Asimismo, se señala que se debe facilitar el acceso equitativo al ROE, y se resalta la complejidad de los procedimientos de coordinación. Por ello, recomienda lo siguiente:

[...] Cuando sea necesaria la coordinación entre países con miras a la utilización de órbitas de satélites, inclusive la órbita de los satélites geoestacionarios, los países interesados deben tener en cuenta el hecho de que el acceso a esa órbita debe realizarse, entre otras cosas, de manera equitativa y de conformidad con el Reglamento de Radiocomunicaciones de la UIT. Por consiguiente, en caso de solicitudes equiparables para acceder al recurso órbita-espectro por parte de un país que ya tenga acceso a dicho recurso, y un país en desarrollo u otro país que trate de acceder a él, el país que ya tenga acceso debe adoptar todas las medidas viables para permitir que el país en desarrollo o el otro país tenga acceso equitativo al recurso órbita-espectro solicitado. (Núm. 8, literal a) 


\section{RÉGIMEN JURÍDICO ESPECIAL}

\section{A. Exposición}

Teniendo en cuenta la importancia que ha devenido la órbita de los satélites geoestacionarios, y con el gran avance que logró el documento anterior en cuanto a la discusión de uso y acceso a la órbita, es menester desarrollar la recomendación presentada para que la UIT pueda entrar a regular el tema y se dé cabal cumplimiento al principio de acceso equitativo que se ha venido proclamando en la normatividad de la Unión Internacional de Telecomunicaciones.

La propuesta toma como punto de partida el punto de vista de Rey Córdoba ante la Subcomisión Jurídica de la Comisión de Utilización del Espacio Ultraterrestre con Fines Pacíficos, aprobada como recomendación en el documento $\mathrm{A}$ / AC.105/738.

\section{B. Desarrollo}

En el numeral 8 se presentan entonces las tres hipótesis sobre el acceso a las posiciones orbitales cuando es necesaria la coordinación entre países, teniendo en cuenta el principio de equidad y la condición de los países en desarrollo, condiciones estipuladas en la Convención de Nairobi de 1982, y en el artículo 44 de la Constitución de la UIT.

La primera y segunda hipótesis aclara que

en caso de solicitudes equiparables para acceder al recurso órbita-espectro por parte de un país que ya tenga acceso a dicho recurso y un país en desarrollo u otro país que trate de acceder a él, el país que ya tenga ese acceso debe adoptar todas las medidas viables para permitir que el país en desarrollo o el otro país tenga acceso equitativo al recurso órbita-espectro solicitado. (Documento A/AC.105/738, anexo III, núm. 8, literal a, 2000)

Es decir que cuando se presente el caso de coordinación de un país que ya accedió con un país que no ha accedido, o de un país desarrollado con un país en vía de desarrollo, debería evitarse el proceso de coordinación y dar registro a la posición orbital al país que no ha accedido o al país en vía de desarrollo.

Por otro lado, se establece el caso que rige actualmente en el sistema de registro del principio de "quien llega primero, tiene prioridad", cuando se presentan casos de coordinación entre países desarrollados:

1. País que ya accedió - país que no ha accedido: Debería tener prelación el país que no ha accedido, sin necesidad de un proceso de coordinación.

2. País en vía de desarrollo - país desarrollado: Debería tener prelación el país en vía de desarrollo, sin necesidad de proceso de coordinación.

3. País desarrollado - país desarrollado: Se le debería seguir dando aplicación al principio de "quien llega primero, tiene prioridad".

No obstante, se pueden encontrar otros casos diferentes a los expuestos en el documento 
en donde un país solicite una posición orbital y deba entrar a coordinar con otros países. Es aquí en donde se encuentra el punto central de mi propuesta, que he querido poner en consideración para que sea desarrollada en la Unión Internacional de Telecomunicaciones en virtud de la Resolución 80 del año 2000, en donde este organismo internacional aboca el mandato de la Subcomisión Jurídica en el documento A/ AC.105/738, anexo III, de desarrollar los principios enunciados en ella:

4. País que ya accedió - país en vía de desarrollo: Debería tener prelación el país en vía de desarrollo, sin necesidad de coordinación. Sin si este país en vía de desarrollo ya accedió a una posición orbital se debería entrar a coordinar bajo el principio de "quien llega primero, tiene prioridad".

5. País que ya accedió - país desarrollado: Se le debería dar aplicación al principio de "quien llega primero, tiene prioridad".

6. País que ya accedió - país que ya accedió: Se le debería dar aplicación al principio de "quien llega primero, tiene prioridad".

7. País que no ha accedido - país en vía de desarrollo: Si el país en vía de desarrollo ya accedió se debe dar prelación al país que no ha accedido. Si el país en vía de desarrollo no ha accedido se debe seguir el procedimiento de coordinación bajo el principio de "quien llega primero, tiene prioridad".

8. País que no ha accedido - país desarrollado: Debería tener prelación el país que no ha accedido, sin necesidad de coordinación.
9. País que no ha accedido - país que no ha accedido: Debería darse el proceso de coordinación bajo el principio de "quien llega primero, tiene prioridad". No obstante, si alguno de los países es un país en vía de desarrollo, debería dársele prioridad a este sin necesidad de coordinación.

10. País en vía de desarrollo - país en vía de desarrollo: Si alguno de los países no ha accedido se le debe dar prioridad a este sin coordinación. De lo contrario se debe entrar a coordinar bajo el principio de "quien llega primero, tiene prioridad".

Es importante precisar algunas cuestiones: cuando el documento A/AC.105/738, anexo III, alude a "solicitudes equiparables", no indica únicamente una misma posición orbital, o una misma frecuencia. Al inicio del proceso de solicitud del ROE ante la UIT, las posiciones y frecuencias pueden generar interferencias a otras que están siendo también solicitadas. Este fue el caso que se presentó en la década del año 1970 entre Colombia, Brasil y Estados Unidos. Tanto Colombia como Brasil se encontraban iniciando el procedimiento de publicación anticipada. A pesar de que estaban solicitando posiciones orbitales diferentes, las transmisiones corrían el riesgo de interferirse. Brasil se opuso entonces a la publicación anticipada hecha por Colombia. Brasil ya contaba con satélites. Colombia no. A esto siguieron aproximadamente tres años de coordinación entre los dos países. Finalmente, Brasil optó por correr la solicitud de posición orbital unos cuantos grados. Estados Unidos también se opuso a la publicación hecha por Colombia pues tenía en órbita un satélite 
muy cerca de donde iba a operar el colombiano. Estados Unidos terminó por correr el satélite en órbita.

El régimen que propongo, en desarrollo del documento citado, pretende evitar estos procesos de coordinación. Así, cuando un país en vía de desarrollo, o un país que no ha accedido a la órbita, solicita el uso de una porción del ROE, esta debería serle adjudicada. Si se causan interferencias con proyectos de satélites, los países desarrollados o con satélites en órbita deberían resolver las interferencias, ya sea, al desplazar la posición orbital, cambiar la forma de la antena o usar frecuencias distintas.

Ahora bien, si las nuevas solicitudes generan interferencias con satélites que ya operan, debe haber un proceso de coordinación, pues los Estados cuyos satélites ya operan, tienen derechos de uso que no pueden ser violados. Es posible, sin embargo, llegar a un acuerdo con el fin de prestar ayuda a países menos favorecidos, como en el caso citado de los Estados Unidos. Si se hubiera recurrido a este régimen especial, por ejemplo, Colombia y Brasil habrían evitado tres años de coordinación, y este se hubiera visto en la obligación de resolver las interferencias posibles, dando prioridad a Colombia en el acceso a la órbita de los satélites geoestacionarios.

\section{Procedimiento}

El procedimiento que debería seguir este régimen especial contaría con tres de los pasos requeridos por el Reglamento de Radiocomunicaciones con la eventualidad de un cuarto paso.
El procedimiento de publicación anticipada debe mantenerse pues permite identificar dónde actuará un nuevo satélite o sistema satelital. Solo en el caso en que se presenten interferencias con un satélite operativo se debe entrar al proceso de coordinación. De lo contrario, se pasará a la notificación y posterior registro de la asignación del ROE para un país en vía de desarrollo o para un país que no haya accedido, según las posibilidades y condiciones expuestas anteriormente. Esto logrará ejecutar a total cabalidad los principios rectores del derecho espacial, así como los atribuidos a la órbita de los satélites geoestacionarios por la Unión Internacional de Telecomunicaciones.

\section{CONCLUSIONES}

Una vez finalizada la exposición de toda la problemática respecto de la órbita de los satélites geoestacionarios y de los esfuerzos que se han presentado para solucionar este problema, podemos concluir varios puntos:

En primer lugar, es de gran importancia recalcar la utilidad para el mundo de las telecomunicaciones que tiene la órbita de los satélites geoestacionarios. Su uso ha revolucionado el mundo, pues permitió la comunicación global con cualquier punto sobre la Tierra. Se deben recordar las características especiales atribuidas por las Naciones Unidas a la órbita de los satélites geoestacionarios, a saber, que es un recurso natural limitado, para ser usado de forma racional, eficaz y económica, para su uso equitativo, en la medida en que son estas quienes permiten regular puntualmente el acceso a ella y su uso. 
La reglamentación internacional que se le ha dado al espacio ultraterrestre se ha generado con miras a proteger su uso de forma que beneficie a toda la humanidad. Sus principios rectores, y en especial para este tema el de "no apropiación del espacio ultraterrestre", son de obligatorio cumplimiento pues permiten un adecuado uso y acceso, teniendo en cuenta que este es considerado como propiedad común de la humanidad.

Es erróneo pensar que las declaraciones de los países ecuatoriales son procedentes, más si se tiene en cuenta la teoría de la obligatoriedad de las normas de derecho espacial, sin importar si se trata de resoluciones o tratados, si fueron firmados o ratificados, porque las decisiones se toman por consenso. La confianza y cooperación que de aquí surgen son indispensables para el funcionamiento internacional del derecho del espacio.

Vemos además que los esfuerzos realizados por el Dr. Alfredo Rey Córdoba en solucionar la problemática originada por Colombia, generó un gran avance sobre la regulación que debería tener, más puntualmente, el acceso y el uso de la órbita en cuestión. Con base en la Resolución 80 de la Unión Internacional de Telecomunicaciones, en la que se aboca el conocimiento del mandato del documento A/AC.105/738, anexo III, se reconoce la importancia y necesidad de un régimen jurídico especial, según se planteó a lo largo de este texto.

En este documento se señaló la necesidad de un régimen jurídico especial, con el fin de resal- tar dos puntos importantes. Primero, se deben dar cumplimiento a los supuestos del uso racional, eficaz y económico que protegen el principio de equidad en cuanto a su acceso. Segundo, debe darse una protección especial a países en situación de desventaja frente a los pioneros en materia espacial.

La órbita de los satélites geoestacionarios es fundamental para el funcionamiento del mundo actual; es necesario que todos los países tengan acceso a ella, una vez reconocidas las desigualdades entre ellos, y el trato diferenciado que por ello debe dárseles. El régimen jurídico especial no solo es importante sino necesario.

\section{Referencias}

Asamblea General de las Naciones Unidas (1969). Convención de Viena sobre el Derecho de los Tratados. Aprobada el 23 de mayo de 1969. Recuperado de http://www.derechos. org/nizkor/ley/viena.html

- (1963). Declaración de los principios jurídicos que deben regir las actividades de los Estados en la exploración y utilización del espacio ultraterrestre. Aprobada por la Asamblea General en su Resolución 1962 (XVIII), de 13 de diciembre de 1963. Recuperado de http://www.oosa.unvienna.org/pdf/publications/ st_space_11rev2S.pdf

- (1967). Tratado sobre los principios que deben regir las actividades de los Estados en la exploración y utilización del espacio ultraterrestre, incluso la Luna y otros cuerpos celes- 
tes. Aprobado por la Asamblea General en su Resolución 2222 (XXI), de 19 de diciembre de 1966.

Biblioteca Salvat de Grandes Temas (1973). Los satélites artificiales. Barcelona: Biblioteca Salvat.

Bohlman, U. (2005). Legal Aspects of the Space Exploration Initiatives. Essential Air and Space Law 2 - Space Law: Current problems and perspectives for future regulation. Utrecht: Eleven International Publishing.

Broomhead, L. y Sassier, D. (1983). A la conquista del espacio. Madrid: EDAF.

Clarke, A. (1945). Extra-terrestrial Relays, Can Rocket Stations Give World-wide Radio Coverage? Recuperado de http://lakdiva.org/ clarke/1945ww/1945ww_oct_305-308.html

Cocca, A. A. (1991). Contribución del consenso al desarrollo progresivo del derecho internacional en las Naciones Unidas. Desarrollo Progresivo del Derecho Internacional. Buenos Aires: Publicaciones de la Fundación Casa de la Cultura de Córdoba.

Conferencia de las Naciones Unidas sobre Organización Internacional (1945). Carta de las Naciones Unidas, firmada el 26 de junio de 1945. Recuperado de http://www.un.org/spanish/aboutun/charter.htm

Constitución Política de Colombia de 1991.
Diccionario de Astronomía. Geoestacionario. Recuperado de http://www.astromia.com/glosario/ geoestacionario.htm

Equatorial Countries. Declaration of the First Meeting of Equatorial Countries, adopted on december 3, 1976. Recuperado de http:// www.jaxa.jp/library/space_law/chapter_2/2-2-12_e.html

Ferrer, E. (1991). Las resoluciones de la asamblea general de las Naciones Unidas como fuente de Derecho Internacional. Desarrollo Progresivo del Derecho Internacional. Buenos Aires: Publicaciones de la Fundación Casa de la Cultura de Córdoba.

Gómez, S. (2013). Los satélites y sus órbitas: Régimen Jurídico del Registro y puesta en órbita de los satélites geoestacionarios (tesis de grado para optar por el título de abogado). Universidad de Los Andes, Bogotá, Colombia.

Instituto Geográfico Agustín Codazzi, Subdirección Cartográfica (1981). Longitudes geográficas de los puntos donde el Ecuador Geográfico corta los límites con Ecuador y Brasil. Documento 5/7786.

Portal del Estado Colombiano. Preguntas y respuestas frecuentes de Espacio Radioeléctrico en Colombia. Recuperado de http://www. gobiernoenlinea.gov.co/web/guest/encyclopedia/-/ wiki/Enciclopedia\%20del\%20Estado/Preguntas+y+ respuestas+frecuentes+de+Espectro+Radioeléctric o+en+Colombia 
Kopal. V. (2009). Tratado sobre los principios que deben regir las actividades de los Estados en la exploración y utilización del espacio ultraterrestre, incluso la Luna y otros cuerpos celestes. United Nations Audiovisual Library of International Law. Recuperado de http:// legal.un.org/avl/pdf/ha/tos/tos_s.pdf

Organización de los Estados Americanos. Decisión 395: Marco regulatorio para la utilización comercial del Recurso Órbita Espectro de los países miembros con el establecimiento, operación, y explotación de sistemas satelitales por parte de las empresas andinas. Sistema de información sobre comercio exterior. Recuperado de http://www.sice.oas. org/trade/junac/decisiones/dec395s.asp

Rincón. C. (2013). Aspectos jurídicos relativos a la utilización del espectro electromagnético y su importancia en las telecomunicaciones por satélite. Revista de Derecho Público, Universidad de los Andes, 30.

Real Academia Española. Diccionario de la lengua española. Recuperado de http://www.rae. es/

Rey Córdoba, A. (1991). El problema de la órbita geoestacionaria en la reforma constitucional. Manuscrito original.

Subcomisión de Asuntos Jurídicos del Comité para la Utilización del Espacio Ultraterrestre con Fines Pacíficos. Algunos aspectos relativos a la utilización de la órbita geoestacionaria. Documento aprobado por la Subcomisión de Asuntos Jurídicos en su 39. ${ }^{\circ}$ período de sesiones (A/AC.105/738, anexo III). Recuperado de http://www.oosa.unvienna.org/pdf/publications/st_space_11rev2S.pdf

Unión Internacional de Telecomunicaciones (1992). Constitución de la Unión Internacional de Telecomunicaciones, aprobada el 22 de diciembre de 1992. Recuperado de http:// www.itu.int/net/about/basic-texts/index.aspx

- (1973). Convenio de Málaga-Torremolinos, aprobado el 25 de octubre de 1973. Recuperado de http://www.itu.int/dms_pub/itu-s/ oth/02/09/s020900000a5201pdfs.pdf

- (1965). Convenio de Montreux, aprobado el 12 de noviembre de 1965. Recuperado de http://www.itu.int/dms_pub/itu-s/oth/02/09/ s02090000095201pdfs.pdf

- (1982). Convenio de Nairobi, aprobado el 6 de noviembre de 1982. Recuperado de http://www.itu.int/dms_pub/itu-s/oth/02/09/ s020900000b5201pdfs.pdf

- (2000). Resolución 80, diligencia debida en la aplicación de los principios constitucionales. Rev.CMR-2000. Recuperado de http://www.itu.int/dms_pub/itu-r/oth/0c/04/ ROC040000070001PDFS.pdf

- (2012). Reglamento de Radiocomunicaciones. 